\title{
The bees of the genus Colletes LATREILLE 1802 of Mongolia (Hymenoptera, Apoidea: Colletidae)
}

With 5 figures

Michael Kuhlmann ${ }^{1}$ and Maxim Yu. Proshchalykin ${ }^{2}$

${ }^{1}$ Department of Life Sciences, The Natural History Museum Cromwell Road, London, SW7 5BD, UK. - m.kuhlmann@nhm.ac.uk

${ }^{2}$ Institute of Biology and Soil Science, Far Eastern Branch of Russian Academy of Sciences, Vladivostok-22 690022, Russia

proshchalikin@biosoil.ru

Published on 2013-12-20

\section{Summary}

All available information about bees of the genus Colletes in Mongolia is summarized. Thirty-three species are currently known from this country. Colletes plumuloides Kuhlmann \& Proshchaly kin sp. n. is described as new from the Dornogovi Aimag. Two species are newly recorded from Mongolia: C. roborovskyi FrIeSE 1913 and C. dubitatus Noskiewicz 1936. The female of C. dubitatus is here described for the first time.

\section{Key words}

Colletes, taxonomy, new species, fauna, Asia, Palaearctic region.

New taxa

Colletes plumuloides Kuhlmann \& Proshchalykin sp. n.

\section{Zusammenfassung}

In der vorliegenden Arbeit werden alle verfügbaren Daten zum Vorkommen der Bienengattung Colletes in der Mongolei zusammengefasst. Derzeit sind 33 Arten bekannt und C. plumuloides Kuhlmann \& Proshchalykin sp. n. wird neu beschrieben basierend auf Material aus dem Dornogovi Aimag. Zwei Arten werden erstmals in der Mongolei nachgewiesen: C. roborovskyi Friese 1913 und C. dubitatus NoskiewiCz 1936. Das Weibchen von C. dubitatus wird hier zum ersten Mal beschrieben. 


\section{Introduction}

The bee genus Colletes comprises about 470 described species with an estimated total of about 700 species (Proshchalykin \& Kuhlmann 2012). It has been recorded from all continents except Antarctica, Australia, Madagascar and Southeast Asia (Michener 2007) and more than 200 species are known from the Palaearctic region with its centre of diversity in Middle Asia (Kuhlmann 2005, 2006). The Colletes bees of Mongolia (Fig. 1) have been studied twice before (KuHLmann \& Dorn 2002, Kuhlmann 2009a). However, taxonomic information about the Mongolian bee fauna is still fragmentary so the additional 560 Colletes specimens recently made available mainly by museum collections in Russia (see Materials and methods) were very welcome. The inadequate state of knowledge is illustrated in this paper by the discovery of another previously undescribed species, new records for the country, the discovery of the hitherto unknown female of C. dubitatus Noskiewicz and the saga regarding the identity of the females of C. edentulus Noskiewicz and C. ravulus Noskiewicz (KuHLmann 2010) that has only recently been solved (Kuhlmann \& Proshchalykin 2011). The bee fauna of Mongolia has an endemic element but can also be seen as a transition from a northern temperate fauna to that of the arid interior of China and Central Asia. Together with the recently studied Colletes fauna of the Asian part of Russia (KuHLMann \& ProshCHALYKIN 2011), investigation of the Mongolian fauna is an important cornerstone for the exploration and a better understanding of the rich but little known Colletes faunas of neighbouring China (KuHLMANN 2002, 2007, Niu et al. 2013) and Central Asia (Kuhlmann 2003, 2005, 2006).

The present study aims to summarize the present state of knowledge of the bee genus Colletes of Mongolia as a basis for further investigations of the largely unexplored but species rich faunas of China and Central Asia. Including the newly studied material the results presented here are based on a total of more than 2600 specimens representing 33 species. Colletes plumuloides Kuhlmann \& Proshchalykin sp. n., and the female of C. dubitatus are here described for the first time.

\section{Materials and methods}

Terminology for the description of species is based on Michener (2007) for general morphology. Puncture density is expressed as the relationship between puncture diameter (d) and the space between them (i), such as $\mathrm{i}=1.5 \mathrm{~d}$ or $\mathrm{i}<\mathrm{d}$. The following abbreviations were used for morphological structures: T-metasomal tergum, S-metasomal sternum, Bl-body length. Measurements follow the guidelines of Michener (2007). Body length was measured from the vertex to the apex of the body. Nomenclature and definition of species groups in Colletes follows Kuhlmann (2000) and Kuhlmann et al. (2009).

Acronyms for collections from which specimens were borrowed or are deposited are as follows:

AMNH American Museum of Natural History, New York, USA (J. Ascher, J. Rozen);

IBSS Institute of Biology and Soil Science, Russian Academy of Sciences, Vladivostok, Russia (A. Lelej);

ISEN Institute of Animal Systematic and Ecology, Russian Academy of Sciences, Novosibirsk, Russia (V. Dubatolov);

OÖLM Oberösterreichisches Landesmuseum/Biology Centre, Linz, Austria (F. Gusenleitner);

PHAS Philadelphia Academy of Sciences, Philadelphia, USA (J. Gelhaus);

PCJS private collection of J. Straka, Prague, Czech Republic;

RCMK research collection of Michael Kuhlmann, London, UK;

ZISP Zoological Institute, Russian Academy of Sciences, St. Petersburg, Russia (S. Belokobylskij, Yu. Astafurova).

The following abbreviations were used for collectors: AT - A. Timokhov, JH - J. Halada, JG - J. Gelhaus, JS - J. Straka, IK - I. Kerzhner, KA - M. Kadlecova, MH - M. Halada, MK - M. Kozlov, YD - Yu. Danilov, VZ - V. Zaitsev.

New distribution records for provinces (Aimags) in Mongolia are marked with an asterisk $\left(^{*}\right)$.

\section{Results}

\section{Species recorded}

\section{Colletes caspicus species-group}

\section{Colletes alini KuHLMANn 2000}

\section{Material examined:}

(2 우 우, $10 o^{\star} o^{\star}$ ): Bayan-Khongor Aimag: $1 \sigma^{\star}, 75 \mathrm{~km} \mathrm{~S}$ Bayankhongor (E100 $\left.53^{\prime} \mathrm{N} 45^{\circ} 31^{\prime}\right), 1500 \mathrm{~m}, 8-9 . \mathrm{VII} .2004$, JS (PCJS); $10^{*}$, Orog-Noor Lake (E100²' N4503'), 15-16. VIII.1967, VZ (ZISP); Usv Aimag: $20^{\top} 0^{\top}$, Togtokhyn-Shil Ridge, 50 km ESE Ulangom (E92 04' N4959'), 7.VIII.1970, IK (ZISP); 3 ๙ $^{\star} \sigma^{\star}, 50 \mathrm{~km}$ E Ulangom (E92 $\left.70^{\circ} \mathrm{N} 49^{\circ} 59^{\prime}\right)$, 10-11.VII.1968, MK (ZISP); Khovd Aimag: $20^{\star} o^{\star}, 20 \mathrm{~km}$ SSW Tsetseg (E9306' N462 ${ }^{\circ}$ ), $2060 \mathrm{~m}, 11 . \mathrm{VI} .2012$, AT (IBSS); Umnugovi Aimag: 2 우, $70 \mathrm{~km} \mathrm{~S} \mathrm{Saynshand}$ (E102 ${ }^{\circ} 33^{\prime}$ N42 55'), 1100 m, 6.VIII.2007, JH (OÖLM); Dornod Aimag: $10^{\star}, 7$ km S Erentsav [= Chuluun-Khorot] (E11480' N4961'), 22.VIII.1975, E. Narchuk (ZISP). 
Published records:

OsYTSHNJuk \& Romankova 1995: 483 (as C. seitzi Alfken 1900); Kuhlmann \& Dorn 2002: 90; ProshCHALYKIN 2007: 879 (as C. seitzi); 2012: 449; KuHLMANN 2009a: 21; Kuhlmann \& ProshChalykin 2011: 7.

Distribution in Mongolia:

Uvs Aimag, ${ }^{\star}$ Bayan-Khongor Aimag, Khovd Aimag, Tuv Aimag, ${ }^{\star}$ Umnugovi Aimag, Dornod Aimag, Sukhbaatar Aimag (Fig. 2).

General distribution:

Russia, Mongolia, North-eastern China.

\section{Colletes squamosus species-group}

\section{Colletes wahrmani NoskIEWICz 1959}

Material examined:

(1 +): Umnugovi Aimag: 1 †, $60 \mathrm{~km}$ E Talyn-Bilgakh-

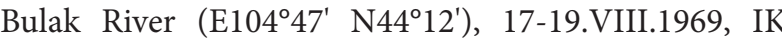
(ZISP).

Published records:

KuHLMANN 2009a: 21; 2009b: 16

Distribution in Mongolia:

Govi-Altai Aimag, ${ }^{\star}$ Umnugovi Aimag (Fig. 2).

\section{General distribution:}

Turkey, Kazakhstan, Turkmenistan, Uzbekistan, Pakistan, Mongolia, China [Xinjiang]

\section{Colletes mixtus species-group}

\section{Colletes dubitatus Noskiewicz 1936}

Material examined:

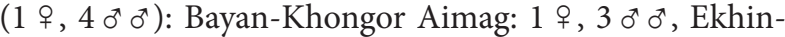
Gool Oasis (E99 ${ }^{\circ} 81^{\prime}$ N42³1'), 11-14.VIII.1969, MK (ZISP/RCMK); Khovd Aimag: 1 ㅇ , 12 km S Altaj (E92 22 N458'), 22.VII.1970, IK (ZISP).

The female of C. dubitatus is here described for the first time. The single female was found with three conspecific males so there is no doubt about its identity.

\section{Diagnosis:}

Colletes dubitatus is morphologically very similar to the other four species (five including the unknown female of C. issykkuli Kunlmann) of this group. The most noticeable character of this species separating it from its relatives are the very broad apical metasomal tergal hair bands that are much broader than the hairless disc of the respective terga (Fig. 4c). In C. kozlovi FrIESE the hairless discs of the metasomal terga are about as broad as the apical hair bands and in C. annejohnae KuHLmann, C. mixtus Radoszkowski and C. stachi Noskiewicz the hairless discs are much broader than the apical hair bands.

\section{Description:}

Female. $\mathrm{Bl}=8.5 \mathrm{~mm}$. Head. Head wider than long. Integument black except mandible dark reddish-brown. Face including clypeus densely covered with long, yellowishwhite, erect hairs (Fig. 4b). Clypeus convex without a longitudinal median depression, supraclypeal area convex in profile. Clypeus very finely and densely punctate; surface between punctures shiny (Fig. 4b). Malar area medially very short, almost linear, finely striate. Antenna black, ventrally dark brown (Fig. 4b).

Mesosoma. Integument black. Mesoscutal disc between punctures smooth and shiny; disc sparsely punctate ( $\mathrm{i}=3.0-4.0 \mathrm{~d})$. Scutellum anteriorly sparsely punctate with more dense punctation apically, surface smooth and shiny. Mesoscutum, scutellum, metanotum, mesepisternum and propodeum densely covered with long yellowish-white erect hairs (Fig. 4a). Wings. Slightly yellowish-brown; wing venation and stigma yellowishbrown. Legs. Integument black to dark reddish-brown. Vestiture whitish, scopa white. Metasoma. Integument black except apical margins of $\mathrm{T}$ yellowish to brownish translucent. T1 in its anterior half densely covered with short appressed hairs leaving a strip of the disc hairless that is narrower than the width of the apical tergal hair band (Fig. 4c); T1 sparsely covered from the front to the disc with long, erect white hairs. T2 - T5 with broad basal and apical hair bands, hairless tergal discs narrower than the width of the apical tergal hair bands (Figs $4 a, c)$. Terga very densely and very finely punctate $(\mathrm{i}<\mathrm{d})$ (Fig. 4c)

\section{Distribution in Mongolia:}

^Bayan-Khongor Aimag, ${ }^{\star}$ Khovd Aimag (Fig. 2).

\section{General distribution:}

Kazakhstan, Turkmenistan, Tajikistan, Uzbekistan, Mongolia.

\section{Colletes kozlovi FrIESE 1913}

\section{Material examined:}

$\left(2 o^{\star} o^{\star}\right)$ : Umnugovi Aimag: $2 \sigma^{\star} o^{\star}, 70 \mathrm{~km} \mathrm{~S}$ Saynshand (E102 $\left.33^{\prime} \mathrm{N}^{\circ} 2^{\circ} 55^{\prime}\right), 1100 \mathrm{~m}, 6 . \mathrm{VII} .2007$, JH (OÖLM).

\section{Published records:}

Noskiewicz 1936: 240; KuHLMANN \& Dorn 2002: 91; KuHLMANN 2005: 1369; 2009a: 21; 2009b: 16; KuHLMANN \& Proshchalykin 2011: 7 
Distribution in Mongolia:

Khovd Aimag, Zavkhan Aimag, Bayan-Khongor Aimag, Umnugovi Aimag, Dornogovi Aimag, Dornod Aimag (Fig. 2).

\section{General distribution:}

Russia, Kazakhstan, Kyrgyzstan, Turkmenistan, Uzbekistan, Mongolia, China (Xinjiang, Gansu, Inner Mongolia).

\section{Colletes mixtus RadoszKowski 1891}

Material examined:

(7 우 우, $\left.27 \sigma^{\top} o^{\top}\right)$ : Bayan-Khongor Aimag: 4 우 우 $6 \mathrm{~m} o^{\top} \#$, Talyn-Bilgekh-Bulak, $13 \mathrm{~km}$ E Tsagan-Bogdo-Ula (E99 ${ }^{\circ} 3^{\prime}$ N42 85'), 16.VIII.1969, MK (ZISP/RCMK); $10^{*}$, Ekhin-Gool Oasis (E99 ${ }^{\circ} 81^{\prime}$ N42 $\left.31^{\prime}\right)$, 31.VIII.1970, MK (ZISP); 3 우 ㅇ, 19 o ๙ $^{*}$, 11-14.VIII.1969, MK (ZISP/

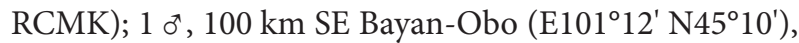
21.VI.1971, MK (ZISP).

Published records:

Kuhlmann \& Dorn 2002: 91; Kuhlmann 2005: 1370.

Distribution in Mongolia:

Khovd Aimag, Govi-Altai Aimag, Bayan-Khongor Aimag (Fig. 3).

\section{General distribution:}

Turkey, Kazakhstan, Tajikistan, Turkmenistan, Uzbekistan, Mongolia, China.

\section{Colletes roborovskyi species-group}

\section{Colletes roborovskyi FrIESE 1913}

\section{Material examined:}

(5 $\left.o^{*} o^{*}\right)$ : Khovd Aimag: $4 o^{\star} o^{\star}, 25 \mathrm{~km}$ N Bulgan, UlyastainGol River (N91 ${ }^{\circ} 85^{\prime}$ N463'), 31.VII.1970, MK (ZISP); Umnugovi Aimag: $10^{\star}, 70 \mathrm{~km} \mathrm{~S}$ Saynshand (E102 ${ }^{\circ} 33^{\prime}$ N4255'), 1100 m, 6.VIII.2007, JH (OÖLM).

Distribution in Mongolia:

${ }^{\star}$ Khovd Aimag, ${ }^{*}$ Umnugovi Aimag (Fig. 2).

\section{General distribution:}

Turkey, Russia, Georgia, Azerbaijan, Kazakhstan, Kyrgyzstan, Tajikistan, Turkmenistan, Mongolia.

\section{Colletes uralensis species-group}

\section{Colletes kaszabi KunLmann 2002}

\section{Material examined:}

(6 우, $8{\sigma^{\top}}^{\top}$ ): Zavkhan Aimag: 1 ㅇ, $40 \mathrm{~km}$ SW Uyasutay, dunes (E96 $\left.31^{\prime} \mathrm{N}^{\circ} 7^{\circ} 31^{\prime}\right)$, 18.VII.2005, KA (OÖLM); Govi-Altai Aimag: $10^{\circ}$, Gobi, Orgon (E98 $\left.23^{\prime} \mathrm{N} 44^{\circ} 43^{\prime}\right)$, 11.VII.2005, P. Tyrner (OÖLM); Bayan-Khongor Aimag:

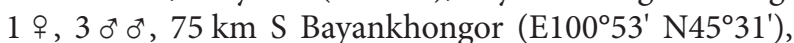
1500 m, 8-9.VII.2004, JS (PCJS/RCMK); Bulgan Aimag: 1 i , $143 \mathrm{~km}$ NE Arvaykheer (E10339' N47²4'), $1300 \mathrm{~m}$, 26.VII.2004, JS (RCMK); Ulaanbaatar: $10^{\top}$ (E10659' N47²5'), VII.1991, M. McKenna (AMNH); Tuv Aimag:

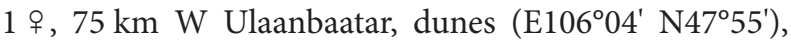
2.VIII.2005, JH (OÖLM); Dornogovi Aimag: 1 o $^{\star}, 2 \mathrm{~km}$ SE Khuvsgol (E109 $\left.70^{\prime} \mathrm{N}^{\circ} 3^{\circ} 50^{\prime}\right)$, 5.VIII.2007, $\mathrm{MH}$ (OÖLM); 2 우, $28 \mathrm{~km}$ SE Chatan-Bulag (E109³0' N430'), 3.VIII.2007, KA (OÖLM); Sukhbaatar Aimag: $10^{\star}, 100 \mathrm{~km}$ SSW Baruun-Urt (E112 $\left.{ }^{\circ} 55^{\prime} \mathrm{N} 45^{\circ} 45^{\prime}\right)$, 1100 m, 30.VII.2007, MH (OÖLM); 1 o $^{\star}, 15$ km SE Khongor, Ongon-Els (E1130' N4572'), 5-6.VII.1971, MK (ZISP).

Published records:

Kuhlmann \& DoRn 2002: 92; Kuhlmann 2009a: 21.

\section{Distribution in Mongolia:}

Uvs Aimag, ${ }^{\star}$ Zavkhan Aimag, Bayan-Khongor Aimag, Govi-Altai Aimag, ${ }^{*}$ Bulgan Aimag, ${ }^{*}$ Ulaanbaatar, Tuv Aimag, Dornogovi Aimag, Dornod Aimag, Sukhbaatar Aimag (Fig. 2).

General distribution:

Mongolia.

\section{Colletes flavicornis species-group}

\section{Colletes emaceatus NoskieWICz 1936}

\section{Material examined:}

(2 우 우, $\left.7 \sigma^{\star} o^{\star}\right)$ : Bayan-Khongor Aimag: $2 \sigma^{\star} o^{\star}, 75 \mathrm{~km} \mathrm{~S}$ Bayankhongor (E100 53' N4531'), 1500 m, 8-9.VII.2004, JS (PCJS); $10^{*}$, Tuin-Gool River, S Bogdo-Ula (E101 ${ }^{\circ} 04^{\prime}$ N4509'), 5.VIII.1969, IK (ZISP); Uvurkhangai Aimag: 1 ㅇ, $2 \sigma^{\star} o^{\star}, 159 \mathrm{~km}$ SW Arvaykheer, $2 \mathrm{~km}$ E Khöövör, Taatsyn Tsagaan Noor, sandy dunes (E101 $\left.{ }^{\circ} 26^{\prime} \mathrm{N} 45^{\circ} 11^{\prime}\right)$, 1250 m, 5.VII.2004, JS (PCJS/RCMK); Umnugovi Aimag: $1 \mathrm{o}^{\top}, 70 \mathrm{~km} \mathrm{~S}$ Saynshand (E102 $\left.33^{\prime} \mathrm{N} 42^{\circ} 55^{\prime}\right), 1100 \mathrm{~m}$, 6.VII.2007, MH (OÖLM); 1 ㅇ, $10^{\top}$, Bordzon-Goby, $80 \mathrm{~km}$ SSE Nomgon (E1057 $\left.{ }^{\circ} \mathrm{N} 42^{\circ} 35^{\prime}\right)$, 5-8.VIII.1967, IK (PCMK).

Published records:

Kuhlmann 2009a: 22. 


\section{Distribution in Mongolia:}

Bayan-Khongor Aimag, Uvurkhangai Aimag, Umnugovi Aimag (Fig. 3).

General distribution:

Kazakhstan, Turkmenistan, Mongolia.

\section{Colletes gusi KunLmann 2009}

Material examined:

(2 우 ㅇ, $2 \diamond^{\star} \diamond^{\star}$ ): Usv Aimag: $1 \diamond^{\star}, 50 \mathrm{~km}$ E Ulangoma, UvsNoor Lake (E92 $\left.{ }^{\circ} 15^{\prime} \mathrm{N} 50^{\circ} 22^{\prime}\right)$, 6.VIII.1970, MK (ZISP); Umnugovi Aimag: $1 \mathrm{o}^{\star}, 70 \mathrm{~km}$ S Saynshand (E102 33 N42 $55^{\prime}$ ), 1100 m, 6.VIII.2007, JH (OÖLM); 1 , 60 km E Talyn-Bilgakh-Bulak River (E104' $45^{\prime}$ N44ํ6'), 14-19. VIII.1969, MK (ZISP); Dornogovi Aimag: 1 ㅇ, 28 km SE Chatan-Bulag (E109³0' N4305'), 3.VIII.2007, KA (OÖLM).

Published records:

KuHLMANN 2009a: 22

Distribution in Mongolia:

${ }^{*}$ Usv Aimag, ${ }^{*}$ Umnugovi Aimag, Dornogovi Aimag, Dornod Aimag (Fig. 3).

General distribution:

Mongolia.

\section{Colletes plumuloides KuHLMANn \& PrOSHCHALYKIN sp. n. $o^{*}$}

Material examined:

$\left(1 \sigma^{\star}\right)$ : Holotype ( $\left.\sigma^{\star}\right)$ : Mongolia, Dornogovi Aimag, $28 \mathrm{~km}$ SE Chatan-Bulag (E109³0' N4305'), 3.VIII.2007, JH (RCMK).

Diagnosis:

Colletes plumuloides belongs to the C. flavicornis speciesgroup and is closely related to C. plumulosus NoskiEwICZ. Both species have a dense crest of short white hairs on the dorsal ridge of the apical part of the gonocoxite and the gonostylus (Fig. 5d) that is unique among the Palaearctic Colletes. In C. plumuloides S7 (Fig. 5c) is of similar shape but only about $2 / 3$ of the size of that of C. plumulosus, the ventral side of the antennal flagellum is orange-brown (Fig. 5a) (blackish-brown in C. plumulosus), malar area shorter, length medially about $1 / 3$ as long as width of mandible base (in C. plumulosus malar area medially about $1 / 2$ as long as width of mandible base) and metasomal tergal hair bands (Fig. 5b) slightly narrower than in C. plumulosus.
Description:

Female. Unknown.

Male. $\mathrm{Bl}=6.0 \mathrm{~mm}$. Head. Head wider than long. Integument black except apical margin of clypeus and most of mandible dark reddish-brown. Face densely covered with long, yellowish-white, erect hairs. Malar area medially about $1 / 3$ as long as width of mandible base, finely striate. Antenna black, ventrally bright orange-brown (Fig. 5a). Mesosoma. Integument black. Mesoscutal disc impunctate, smooth and shiny. Scutellum almost impunctate, only lateral and apical margins with some small punctures, surface smooth and shiny. Mesoscutum, scutellum, metanotum, mesepisternum and propodeum covered with long, greyish-white erect hairs (Fig. 5a). Wings. Slightly yellowish-brown; wing venation and stigma yellowish-brown. Legs. Integument mostly black, basal and apical ends of tibiae and tarsi reddish-brown. Vestiture white. Metasoma. Integument black except apical tergal depressions yellowish translucent (Fig. 5b). Tergal hairs are rubbed off in this single specimen so little can be said about pilosity patterns but apical tergal hair bands are probably broad (Fig. 5b). Terga densely and finely punctate $(\mathrm{i}=0.5-1.0 \mathrm{~d})$, between punctures smooth and shiny (Fig. 5b). Terminalia. Genitalia and S7 as illustrated (Figs $5 c, d$ ).

Etymology:

This species is named for its similarity to C. plumulosus.

General distribution:

Only known from the type locality in Mongolia (Fig. 3).

Floral hosts:

unknown.

Seasonal activity (first-last observations). VIII.

\section{Colletes popovi NoskIEWICz 1936}

Material examined:

(1 우 $\left.3 \sigma^{\star} o^{\star}\right)$ : Khovd Aimag: $1 \sigma^{\star}, 12 \mathrm{~km}$ SW Altaj-som, Bodonchin-Gol River (E92 ${ }^{\circ} 5^{\prime}$ N45 $\left.{ }^{\circ} 81^{\prime}\right)$, 22.VII.1970, MK (ZISP); Govi-Altai Aimag: $10^{\star}, 60 \mathrm{~km}$ SW Tsel (E9508' N4542'), 19.VII.1970, VZ (ZISP); Bayan-Khongor Aimag: $1 \sigma^{\star}$, Talyn-Bilgekh-Bulak, $13 \mathrm{~km}$ E TsaganBogdo-Ula (E99 ${ }^{\circ} 3^{\prime}$ N4285'), 16.VIII.1969, MK (ZISP); Uvurkhangai Aimag: 1 , Tatsyn-Tsagan-Noor Lake

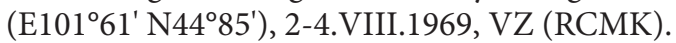

Published records:

KuHLMAnN 2005: 1372.

Distribution in Mongolia:

*Khovd Aimag, ${ }^{*}$ Govi-Altai Aimag, ${ }^{*}$ Bayan-Khongor Aimag, ${ }^{\star}$ Uvurkhangai Aimag (Fig. 3). 
General distribution:

Kazakhstan, Mongolia, China.

\section{Colletes fodiens species-group}

\section{Colletes daviesanus SMıтH 1846}

Material examined:

$\left(1{\sigma^{\top}}^{\top}\right)$ : Umnugovi Aimag: $1 \sigma^{\top} \sigma^{*}, 65 \mathrm{~km}$ SE Nomgon (E105이' N42³5'), 9.VIII.1967, IK (ZISP).

Published records:

Kuhlmann \& Dorn 2002: 93; Kuhlmann 2005: 1373; 2009a: 24; Proshchalykin \& Kuhlmann 2012: 15; PROSHCHALYKIN 2012: 449.

Distribution in Mongolia:

Uvs Aimag, Zavkhan Aimag, Tuv Aimag, ${ }^{\star}$ Umnugovi Aimag (Fig. 3).

General distribution:

Ireland, Great Britain, Norway, Sweden, Netherlands, Belgium, France, Germany, Switzerland, Liechtenstein, Austria, Italy, Czech Republic, Slovakia, Hungary, Slovenia, Croatia, Serbia, Finland, Poland, Estonia, Romania, Ukraine, Russia, Turkey, Georgia, Armenia, Kazakhstan, Kyrgyzstan, Mongolia, North-eastern China.

\section{Colletes edentuloides KunLmann 2011}

Material examined:

(11 우 우): Tuv Aimag: 11 웅, $50 \mathrm{~km} \mathrm{~N}$ Ulaanbaatar

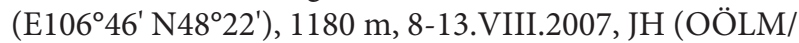
RCMK).

Published records:

Kuhlmann \& Proshchalykin 2011: 24.

Distribution in Mongolia:

Tuv Aimag (Fig. 3).

General distribution:

Mongolia.

\section{Colletes edentulus NoskIEWICZ 1936}

Published records:

Kuhlmann \& Dorn 2002: 94; Kunlmann 2009a: 25.

Distribution in Mongolia:

Uvs Aimag, Bayan-Khongor Aimag, Tuv Aimag (Fig. 3).

\section{General distribution:}

Turkey, Azerbaijan, Mongolia.

\section{Colletes fodiens (Fourcroy 1785)}

Published records:

Kuhlmann \& Dorn 2002: 94 (as C. fodiens kirgisicus Radoszkowski 1868); Kunlmann 2005: 1373 (as C. fodiens kirgisicus); 2009b: 17 (C. fodiens kirgisicus); Kuhlmann \& Proshchalykin 2011: 9; РroshchaLYKIN \& KuHLMANN 2012: 16.

Distribution in Mongolia:

Uvs Aimag (Fig. 3).

\section{General distribution:}

Great Britain, Portugal, Spain, Sweden, Netherlands, Belgium, France, Denmark, Germany, Switzerland, Austria, Finland, Estonia, Latvia, Lithuania, Poland, Czech Republic, Slovakia, Hungary, Italy, Slovenia, Croatia, Serbia, Romania, Bulgaria, Ukraine, Russia, Turkey, Azerbaijan, Kazakhstan, Kyrgyzstan, Iran, Pakistan, Mongolia, China [Xinjiang].

\section{Colletes inexpectatus NoskieWICz 1936}

Published records:

Kunlmann \& Dorn 2002: 94; Kunlmann 2005: 1373.

Distribution in Mongolia:

Uvs Aimag (Fig. 2).

General distribution:

Austria, Poland, Czech, Slovakia, Hungary, Yugoslavia, Bulgaria, Ukraine, Kazakhstan, Kyrgyzstan, Tajikistan, Turkmenistan, Uzbekistan, Mongolia.

\section{Colletes ravulus NoskIEWICz 1936}

Material examined:

(2우우, $\left.2 \sigma^{\top} \sigma^{\top}\right)$ : Usv Aimag: 2 우우, channel between Airag and Khirgis-Noor (E93 $\left.20^{\prime} \mathrm{N} 49^{\circ} 10^{\prime}\right)$, 28-29. VIII.1968, MK (ZISP/RCMK); Dornod Aimag: 1 ㅇ, $10^{\circ}$,

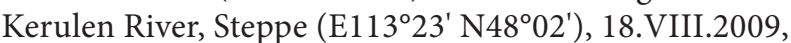
YD (ISEN/RCMK); $10^{\top}$, Choibalsan, Steppe (E114 $27^{\prime}$ N4830'), 20.VIII.2009, YD (ISEN).

Published records:

Kuhlmann \& Dorn 2002: 94; Kuhlmann \& ProshchaLYKIN 2011: 10. 
Distribution in Mongolia:

Usv Aimag, Dornod Aimag (Fig. 2).

General distribution:

Russia, Mongolia, China [Inner Mongolia].

\section{Colletes cunicularius species-group}

Colletes cunicularius (LINNAEUs 1761)

Published records:

Kuhlmann \& Dorn 2002: 94; Kuhlmann \& ProshchaLYKIN 2011: 14; ProshChalykin \& Kuhlmann 2012: 24; ProshCHALYKIN 2012: 449.

Distribution in Mongolia:

Khovd Aimag (Fig. 2).

\section{General distribution:}

Ireland, Great Britain, Spain, Norway, Sweden, Netherlands, Belgium, France, Denmark, Germany, Switzerland, Austria, Finland, Poland, Czech Republic, Slovakia, Hungary, Italy, Slovenia, Serbia, Romania, Greece, Ukraine, Russia, Turkey, Mongolia, North-eastern China.

\section{Colletes foveolaris species-group}

\section{Colletes reinigi NoSKIEWICZ 1936}

Material examined:

$\left(1 \sigma^{*}\right)$ : Tuv Aimag: $1 \sigma^{\star}, 15 \mathrm{~km}$ S Ulaanbataar, Zuunmod

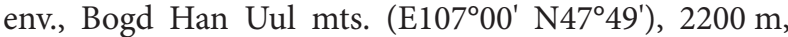
29.VII.2002, JS (RCMK).

Published records:

KuHLMAnN 2009a: 25.

Distribution in Mongolia:

Arkhangai Aimag, ${ }^{\star}$ Tuv Aimag, Umnugovi Aimag (Fig. 2).

\section{General distribution:}

Tajikistan, Mongolia, China.

Remarks:

Kuhlmann (2009) reported C. cf. reinigi for Mongolia for the first time based on male specimens but some uncertainty remained about the identity of the specimens. In the meantime males identified by Noskiewicz could be studied confirming the previous identification.
Colletes clypearis species-group

\section{Colletes cinerascens MorawITZ 1894}

Material examined:

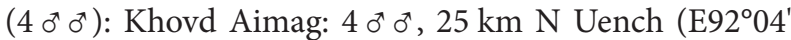
N46 $\left.{ }^{\circ} 5^{\prime}\right), 1700$ m, 15.VI.2012, AT (IBSS).

Published records:

Kuhlmann \& Dorn 2002: 95; Kuhlmann 2005: 1377; 2009a: 25; 2009b: 18

Distribution in Mongolia:

Uvs Aimag, ${ }^{\star}$ Khovd Aimag, Govi-Altai Aimag, BayanKhongor Aimag (Fig. 2).

General distribution:

Kyrgyzstan, Tajikistan, Kazakhstan, Mongolia.

\section{Colletes ebmeri KunLMAnn 2002}

Material examined:

( 1 ㅇ , $4 \sigma^{\top} \sigma^{\top}$ ): Khovd Aimag: 1 ㅇ, $40 \mathrm{~km} \mathrm{~N}$ Uench-somona (E92 ${ }^{\circ} 06^{\prime}$ N463'), 31.VII-1.VIII.1968, MK (ZISP); Govi-Altai Aimag: $1 \sigma^{\star}, 37 \mathrm{~km}$ NE Tsogt, Dutiin-Daba Ridge (E97 $\left.{ }^{\circ} 13^{\prime} \mathrm{N}^{\circ} 5^{\circ} 67^{\prime}\right)$, 14.VII.1970, IK (ZISP); Khuvsgul Aimag: $1 \mathrm{o}^{*}$, Renchinihumbe Soum, $19 \mathrm{~km}$ N Renchinihumbe (E99 $\left.43^{\prime} \mathrm{N}^{\circ} 1^{\circ} 16^{\prime}\right), 1595 \mathrm{~m}, 2$.VII.2006, JG (PHAS); $10^{\star}, 20 \mathrm{~km}$ SW Buren-Khan (E99 ${ }^{\circ} 85^{\prime}$ N49 $\left.{ }^{\circ} 51^{\prime}\right)$, 30.VI.1968, MK (PCMK); Ulaanbaatar: $10^{*}$ (E106 59' N474'), 11.VI.1967, IK (ZISP).

Published records:

Kuhlmann \& Dorn 2002: 95; Kuhlmann 2009a: 25.

Distribution in Mongolia:

*Khovd Aimag, ${ }^{\star}$ Gobi-Altai Aimag, ${ }^{\star}$ Khuvsgul Aimag, Arkhangai Aimag, Bayan-Khongor Aimag, ${ }^{\star}$ Ulaanbaatar (Fig. 2).

General distribution:

Mongolia.

\section{Colletes floralis EvERSMANn 1852}

\section{Material examined:}

$\left(15 \sigma^{\star} \sigma^{\top}\right)$ : Khuvsgul Aimag: $1 \sigma^{\star}$, Ongonii davas (mountain pass), $12 \mathrm{~km}$ E Tsagaan-Uur (E101 $\left.{ }^{\circ} 42^{\prime} \mathrm{N} 50^{\circ} 33^{\prime}\right)$, 1273 m, 16.VII.2005, JG (PHAS); 3 đ $^{\star}$, ChandmaniOndor Soum, unnamed trib. of Hohoo Gol (E100 $44^{\prime}$ N5040'), 1566 m, 18.VII.2005, JG (PHAS); Arkhangai Aimag: $1 \sigma^{\star}$, Tsenkher Soum, Nuurlin Khooloi lake, $9 \mathrm{~km} \mathrm{SW}$ of Tavanbulag (E101 $\left.{ }^{\circ} 49^{\prime} \mathrm{N} 47^{\circ} 20^{\prime}\right), 1693 \mathrm{~m}$, 10.VII.2004, JG (PHAS); Bulgan Aimag: $10^{\star}$, Teshig 
Soum, main branch of Tariankhtain Gol (E10306 N4942'), 911 m, 10.VII.2005, JG (PHAS); Uvurkhangai Aimag: $1 \sigma^{\star}$, Kharkhorin Soum, Sant Uul pass, $23 \mathrm{~km} \mathrm{SE}$ Shinebrigade (E103요' N46 $\left.{ }^{\circ} 2^{\prime}\right)$, 5.VII.2007, JG (PHAS); $10^{\star}$, Mongol Els n. res., dunes (E10339 N47²4), 1320 m, 31.VII.2005, KA (RCMK); Selenge Aimag: $10^{\star}$, Khuder Soum, unnamed stream, 54,2 km E Tavin (E107 16 N4946'), 907 m, 25.VII.2003, JG (PHAS); Ulaanbaatar: $2 \sigma^{\top} \sigma^{\star}$, Zuunmod env. (E106 $\left.59^{\prime} \mathrm{N}^{\circ} 7^{\circ} 45^{\prime}\right), 1630 \mathrm{~m}$, 27.VII.2004, JS (OÖLM); 2 o $^{\top}$ o $^{\star}$ (E106 $59^{\prime}$ N47 $\left.45^{\prime}\right)$, 14.VII.1967, VZ (ZISP); Tuv Aimag: $10^{\top}$, Batsumber Soum, Haraa Gol, $5 \mathrm{~km}$ Bayanbuural (E106 $\left.47^{\prime} \mathrm{N} 48^{\circ} 30^{\prime}\right)$, $1051 \mathrm{~m}, 16$. VII.2003, JG (PHAS); 1 ๙ $^{\star} 15 \mathrm{~km} \mathrm{~S} \mathrm{Ulaan-}$ bataar, Zuunmod env., Bogd Han Uul mts. (E107 00 N47²49'), 2200 m, 29.VII.2002, JS (PCJS).

Published records:

Kuhlmann \& Dorn 2002: 96; Kuhlmann 2005: 1377; 2009a: 28; 2009b: 18; ProshCHALYKIn 2007: 878; 2012: 449; Kuhlmann \& Proshchalykin 2011: 15.

\section{Distribution in Mongolia:}

${ }^{*}$ Khuvsgul Aimag, Arkhangai Aimag, Bayan-Khongor Aimag, ${ }^{\star}$ Bulgan Aimag, ${ }^{\star}$ Uvurkhangai Aimag, ${ }^{\star}$ Selenge Aimag, Ulaanbaatar, Tuv Aimag (Fig. 2).

\section{General distribution:}

Spain, Ireland, Great Britain, France, Norway, Sweden, Germany, Switzerland, Austria, Italy, Finland, Poland, Czech Republic, Hungary, Greece, Turkey, Belarus, Russia, Georgia, Kazakhstan, Kyrgyzstan, Tajikistan, Iran, Mongolia, India, Japan.

\section{Colletes fulvicornis NoSKIEWICz 1936}

\section{Material examined:}

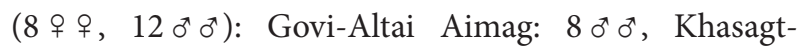
Khairkhan Ridge, 17 km S Dzhargalan (E95 $54^{\prime}$ N4650'), 1600-2100 m, 5-6.VI.2012, AT (IBSS); Bayan-Khongor Aimag: 1 \% , 86 km NW Bayankhongor, along Ölziyt Gol (E10004' N4650'), 2070 m, 14.VII.2004, JS (OÖLM); 1 †, $56 \mathrm{~km} \mathrm{NW}$ Bayankhongor, south slope (E100 ${ }^{\circ} 12^{\prime}$ N463'), $2220 \mathrm{~m}, 11-12 . \mathrm{VII} .2004$, JS (RCMK); 1 ㅇ, $75 \mathrm{~km} \mathrm{~S}$ Bayankhongor (E10053' N4531'), $1500 \mathrm{~m}, 8-9$. VII.2004, JS (PCJS); Uvurkhangai Aimag: 1 ㅇ, $12 \mathrm{~km}$ SW Araykheer, Ongij-Gol River (E102 $\left.{ }^{\circ} 49^{\prime} \mathrm{N} 46^{\circ} 22^{\prime}\right)$, 1770 m, 3.VII.2004, JS (OÖLM); Tuv Aimag: 3 우 우, $15 \mathrm{~km}$ S Ulaanbataar, Zuunmod env., Bogd Han Uul mts. (E10700' N47²9'), 2200 m, 29.VII.2002, JS (PCJS); Umnugovi Aimag: $3 \sigma^{\top} \sigma^{\star}$, Dalan-Dzadgad, Yolyn Am canyon, Covi Curvan Sayhan National Park (E104 04 N4329'), 2240 m, 24.VII.2002, JS (PCJS); 1 ㅇ, GurvanSaikhan, $40 \mathrm{~km} \mathrm{~S}$ Bulgan (E103 $\left.87^{\prime} \mathrm{N} 43^{\circ} 78^{\prime}\right)$, 28-29. VII.1967, IK (ZISP); Khentii Aimag: $1 \sigma^{\star}, 12$ km N Gal-

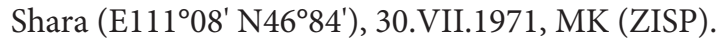

Published records:

Osytshnjuk \& Romankova 1995: 483; Kuhlmann \& Dorn 2002: 96; Kuhlmann 2009a: 28; Kuhlmann \& ProshCHALYKIN 2011: 16.

\section{Distribution in Mongolia:}

Uvs Aimag, Khovd Aimag, Govi-Altai Aimag, Arkhangai Aimag, Bayan-Khongor Aimag, Uvurkhangai Aimag, Ulaanbaatar, Tuv Aimag, Dundgovi Aimag, ${ }^{\star}$ Umnugovi Aimag, Khentii Aimag, Dornod Aimag (Fig. 2).

\section{General distribution:}

Russia, Mongolia, North-eastern China.

\section{Colletes impunctatus NyLANDER 1852}

\section{Material examined:}

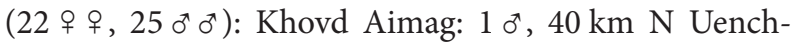
Somon (E92 ${ }^{\circ} 06^{\prime}$ N4638'), 31.VII-1.VIII.1968, MK (ZISP); Zavkhan Aimag: $3 \sigma^{\top} \sigma^{\top}, 40 \mathrm{~km}$ SW Uyasutay, dunes (E96 $31^{\prime}$ N4731'), 18.VII.2005, KA, JH (OÖLM); $10^{\star}, 30 \mathrm{~km}$ WNW Tes-Somon (E96 $\left.{ }^{\circ} 13^{\prime} \mathrm{N} 48^{\circ} 25^{\prime}\right), 3-4$. VII.1968, MK (ZISP); Govi-Altai Aimag: $10^{\star}$, KhasagtKhairkhan Ridge, 17 km S Dzhargalan (E95 ${ }^{\circ} 54^{\prime}$ N46 50 '), 1600-2100 m, 5.VI.2012, AT (IBSS); Khuvsgul Aimag: $1 \sigma^{\top}$, Renchinihumbe Soum, $19 \mathrm{~km}$ N Renchinihumbe (E99 ${ }^{\circ} 43^{\prime}$ N51 $\left.{ }^{\circ} 16^{\prime}\right), 1595$ m, 2.VII.2006, JG (PHAS); 1 ơ $^{\star}$, Renchinihumbe Soum, Hog Gol River, 25 km W Renchinihumbe (E99 $\left.19^{\prime} \mathrm{N} 51^{\circ} 06^{\prime}\right), 1569 \mathrm{~m}, 1 . \mathrm{VII} .2006$, JG (PHAS); $10^{*}$, Chandmani-Ondor Soum, Hohoo Gol, $1.5 \mathrm{~km}$ SE Chandmani-Ondor (E100 $57^{\prime}$ N50 $\left.28^{\prime}\right)$, $1251 \mathrm{~m}, 18 . \mathrm{VII} .2005$, JG (PHAS); $20^{\star} \mathrm{o}^{\star}$, Delger-Muren River, Buren-Khan (E99 ${ }^{\circ} 2^{\prime}$ N50²1'), 28-29.VI.1968, MK (ZISP); Arkhangai Aimag: $2 \sigma^{\star} \sigma^{*}$, Tariat Soum, Terkhiin Tsagan Nuur, $8 \mathrm{~km}$ W Khorgo/TariatNuurlin Khooloi lake, $9 \mathrm{~km}$ SW Tavanbulag (E99 $\left.{ }^{\circ} 47^{\prime} \mathrm{N} 48^{\circ} 09^{\prime}\right)$, 2057 m, 18.VII.2004, JG (PHAS); 7 ㅇ , 4 o $^{\star}$, ChulutynGol River (E100¹9' N47²4'), 1940 m, 23.VII.2005, JH (OÖLM); $10^{\star}$, Khangaun Mts., 5 km N Khunt (E99 $08^{\prime}$ N475'), 21.VII.2005, JH (OÖLM); $10^{\star}$, ChulutynGol River, Chulut (E100¹8' N47º6'), 29.VI.1975, MK (ZISP); 7 우 우, 1 o $^{\star}, 145 \mathrm{~km} \mathrm{NW}$ Tsetserleg, Tariat env., Horgo Terhiyn Tsagaan Nuur National Park (E99 $51^{\prime}$ N48 ${ }^{\circ} 11^{\prime}$ ), $2160 \mathrm{~m}, 17-18$. VII.2002, JS (PCJS); Uvurkhangai Aimag: $10^{\star}$, Mongol Els, dunes (E103 ${ }^{\circ} 39^{\prime}$ N47 $24^{\prime}$ ), 1320 m, 31.VII.2005, KA (OÖLM); Ulaanbaatar: 1 ㅇ, Zuunmod env. (E106 $\left.59^{\prime} \mathrm{N} 47^{\circ} 45^{\prime}\right), 1630 \mathrm{~m}$, 27.VII.2004, JS (OÖLM); Tuv Aimag: 3 ㅇ 우, $50 \mathrm{~km} \mathrm{~N}$ Ulaanbaatar (E106 $46^{\prime} \mathrm{N}^{\circ} 8^{\circ} 22^{\prime}$ ), $1180 \mathrm{~m}, 8$ 8-13.VIII.2007, JH (OÖLM); 1 ㅇ, Gobi-Terelj National Park, 30 km E Ulaanbataar,Tereli env. (E107 $\left.24^{\prime} \mathrm{N}^{\circ} 7^{\circ} 50^{\prime}\right), 1420 \mathrm{~m}$, 13.VII.2002, JS (PCJS); 1 ㅇ, $15 \mathrm{~km} \mathrm{~S} \mathrm{Ulaanbataar} \mathrm{at}$ Zuunmod env., Bogd Han Uul mts. (E107 $\left.00^{\prime} \mathrm{N} 47^{\circ} 49^{\prime}\right)$, 2200 m, 29.VII.2002, JS (PCJS); 1 ㅇ, Ulaanbaatar, Tola

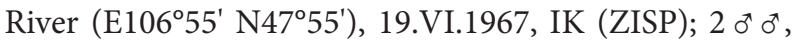
Mungen-Mor't (E108²' N48¹7'), 6-7.VIII.1967, MK 
(ZISP); Umnugovi Aimag: $2{\sigma^{\star} \sigma^{\star}}$, Dalan-Dzadgad, Yolyn Am canyon, Covi Curvan Sayhan National Park (E10404' N4329'), $2240 \mathrm{~m}, 24 . \mathrm{VII} .2002$, JS (PCJS); Dornod Aimag: 1 ㅇ, $50 \mathrm{~km}$ SW Choibalsan (E114 ${ }^{\circ} 10^{\prime}$ N47ㅇ' $), 960$ m, 25.VII.2007, JH (OÖLM).

Published records:

Kuhlmann \& Dorn 2002: 97; Proshchalykin 2007: 879; 2012: 450; Kuhlmann 2009a: 28; Kuhlmann \& PROSHCHALYKIN 2011: 17.

Distribution in Mongolia:

Bayan-Ulgii Aimag, Uvs Aimag, Khovd Aimag, Zavkhan Aimag, Govi-Altai Aimag, Khuvsgul Aimag, Arkhangai Aimag, Bayan-Khongor Aimag, Uvurkhangai Aimag, Ulaanbaatar, Tuv Aimag, ${ }^{\star}$ Dornod Aimag, ${ }^{\star}$ Umnugovi Aimag (Fig. 2).

\section{General distribution:}

Norway, Sweden, Netherlands, France, Denmark, Germany, Switzerland, Austria, Finland, Poland, Czech Republic, Italy, Russia, Mongolia.

\section{Colletes jankowskyi RADoszKowsKI 1891}

Material examined:

(12 우 우, $\left.2 \sigma^{\star} \sigma^{\star}\right)$ : Arkhangai Aimag: 2 우 우, $2 \sigma^{\star} o^{\star}, 90 \mathrm{~km}$ NE Tsetserleg (E102 $\left.25^{\prime} \mathrm{N} 48^{\circ} 08^{\prime}\right), 1940$ m, 24.VII.2004, JS (OÖLM); 1 ㅇ, Khangaun Mts., 5 km N Khunt (E99 $08^{\circ}$ N47 $58^{\prime}$ ), 20.VII.2005, JH (OÖLM); 1 क , $25 \mathrm{~km} \mathrm{NE}$ Tsetserleg (E101 $\left.{ }^{\circ} 45^{\prime} \mathrm{N} 47^{\circ} 38^{\prime}\right), 1730 \mathrm{~m}, 23 . \mathrm{VII} .2004$, JS (OÖLM); Bayan-Khongor Aimag: 1 ㅇ, 86 km NW Bayankhongor, along Ölziyt Gol (E1000' N4650'), 2070 m, 14.VII.2004, JS (OÖLM); Uvurkhangai Aimag: 1 ㅇ , ArtsBogdo Ridge, $20 \mathrm{~km} \mathrm{~S}$ Khovd (E102 $\left.{ }^{\circ} 15^{\prime} \mathrm{N} 44^{\circ} 43^{\prime}\right), 12-13$. VIII.1967, VZ (ZISP); Ulaanbaatar: 4 우 우, Zuunmod env. (E106 $\left.{ }^{\circ} 59^{\prime} \mathrm{N} 47^{\circ} 45^{\prime}\right), 1630 \mathrm{~m}, 27 . V I I .2004$ JS (OÖLM); Tuv Aimag: 1 ㅇ, $75 \mathrm{~km}$ W Ulaanbaatar, dunes (E106ํำ N47 55'), 2.VIII.2005, JH (OÖLM); 1 , 50 km N Ulaanbaatar (E106 $\left.46^{\prime} \mathrm{N} 48^{\circ} 22^{\prime}\right), 1180 \mathrm{~m}, 8-13$.VIII.2007, JH (OÖLM).

Published records:

Osytshnjuk \& Romankova 1995: 484; Kuhlmann \& Dorn 2002: 98; KuHLMAnn 2009a: 29; 2009b: 18; KuHLMANN \& ProshChalykin 2011: 17; Proshchalykin 2012: 450

\section{Distribution in Mongolia:}

Uvs Aimag, Arkhangai Aimag, *Bayan-Khongor Aimag, ${ }^{*}$ Uvurkhangai Aimag, Ulaanbaatar, Tuv Aimag (Fig. 2).

\section{General distribution:}

Russia, Tajikistan, Mongolia, Western and North-eastern China.

\section{Colletes pseudocinerascens NoskiewICz 1936}

Material examined:

(2 우 우, $5 \sigma^{\star} \sigma^{\star}$ ): Usv Aimag: $3 \sigma^{\star} \sigma^{\star}$, Togtokhyn-Shil Ridge, $50 \mathrm{~km}$ ESE Ulangom (E92 ${ }^{\circ} 15^{\prime}$ N50²2'), 7.VIII.1970, MK (ZISP); Zavkhan Aimag: $10^{\star}, 30 \mathrm{~km}$ WNW Tes-Somon (E96 ${ }^{\circ} 13^{\prime}$ N4825'), 3-4.VII.1968, MK (ZISP); Khuvsgul Aimag: 2 우, $10^{\star}, 25 \mathrm{~km}$ NNE Ulan-Ula (E1150. N4910'), 15-16.VII.1975, MK (ZISP).

Published records:

Kuhlmann \& Dorn 2002: 98; Kuhlmann 2009a: 29; KuHLMann \& ProshChalykin 2011: 18

\section{Distribution in Mongolia:}

Usv Aimag, Zavkhan Aimag, ${ }^{\star}$ Khuvsgul Aimag (Fig. 3).

\section{General distribution:}

Russia, Mongolia.

\section{Colletes sidemii RadoszKowski 1891}

Material examined:

(13 우 우, $\left.250 o^{\star} o^{\star}\right)$ : Usv Aimag: $1 \sigma^{\star}, 5 \mathrm{~km}$ S KhyargasNoor (E93 $\left.{ }^{\circ} 20^{\prime} \mathrm{N}^{\circ} 9^{\circ} 10^{\prime}\right), 10 . \mathrm{VIII} .1970, \mathrm{VZ}$ (ZISP);

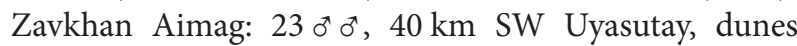
(E96 $\left.{ }^{\circ} 31^{\prime} \mathrm{N}^{\circ} 7^{\circ} 31^{\prime}\right)$, 18.VII.2005, KA, JH (OÖLM); Khuvsgul Aimag: $1 \mathrm{o}^{\star}$, Erdenebulgan Soum, $12.6 \mathrm{~km}$ S Erdenebulgan (E101³7' N4959'), 1344 m, 14.VII.2005, JG (PHAS); $10^{\star}$, Bayanzurkh Soum, Delger Moron Gol, $8.3 \mathrm{~km} \mathrm{~W}$ Bayanzurkh (E98 ${ }^{\circ} 51^{\prime}$ N50 $\left.10^{\prime}\right), 1580 \mathrm{~m}$, 22.VII.2005, JG (PHAS); $20^{\star} 0^{\star}$, Ider River, Dzhargalant (E1008' N4879'), 19-20.VII.1975, MK (ZISP); $10^{\star}$, Delger-Muren River, Buren-Khan (E99 02' N50²1'), 28-29. VI.1968, MK (ZISP); Arkhangai Aimag: 1 ㅇ, $30^{\star} \sigma^{\star}$, Chuluut Soum, Chuluutin Gol, $33 \mathrm{~km}$ SW Chuluut/Jargalant

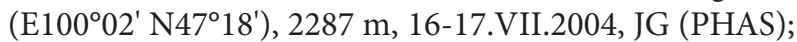
$20^{\top} 0^{\top}$, Tsenkher Soum, Nuurlin Khooloi lake, 9 km SW Tavanbulag (E101 ${ }^{\circ} 49^{\prime}$ N47²0'), 1693 m, 10.VII.2004, JG (PHAS); $64 o^{\star} 0^{\star}$, Khangaun Mts., 5 km N Khunt (E99 $08^{\prime}$ N47 $\left.58^{\prime}\right), 21 . V I I .2005, \mathrm{KA}, \mathrm{JH}$ (OÖLM); $4 \sigma^{\star} \sigma^{\star}, 100 \mathrm{~km}$ NW Tsetserleg, Chulutyn-Gol (E100 ${ }^{\circ} 19^{\prime}$ N47 $\left.48^{\prime}\right)$,

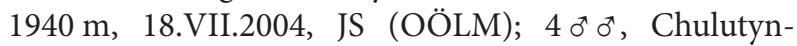
Gol River (E100 $\left.{ }^{\circ} 19^{\prime} \mathrm{N} 47^{\circ} 48^{\prime}\right), 1940 \mathrm{~m}, 23$.VII.2005, JH

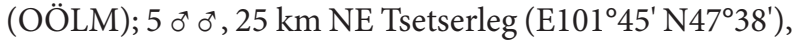
$1730 \mathrm{~m}, 23$. VII.2004, JS (OÖLM); 6 우 ㅇ, $23 o^{\star} 0^{\star}, 90 \mathrm{~km}$ NE Tsetserleg (E102 $\left.2^{\circ} 5^{\prime} \mathrm{N} 47^{\circ} 03^{\prime}\right), 1400 \mathrm{~m}, 27 . \mathrm{VII} .2005$, KA, JH (OÖLM); $350^{\top} 0^{\star}$, Tsagan-Noor Lake (E99 ${ }^{\circ} 43^{\prime}$ N48 ${ }^{\circ} 11^{\prime}$ ), 2100 m, 22.VII.2005, JH (OÖLM); $450^{\star} o^{\star}$, Khangayn Mts., $30 \mathrm{~km} \mathrm{~S}$ Khunt (E99 $\left.08^{\prime} \mathrm{N} 47^{\circ} 58^{\prime}\right)$, 20.VII.2005, KA, JH (OÖLM); 3 우 우 $145 \mathrm{~km} \mathrm{NW}$ of Tsetserleg, Tariat env., Horgo Terhiyn Tsagaan Nuur National Park (E99 ${ }^{\circ} 1^{\prime}$ N48 $\left.{ }^{\circ} 11^{\prime}\right), 2160$ m, 17.VII.2002,

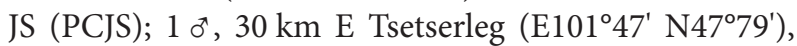
1.VII.1975, MK (ZISP); Uvurkhangai Aimag: $20^{\star} 0^{\star}$, Hujirt Soum, Khavtsaliin Gol valley, Pond (E10302' 


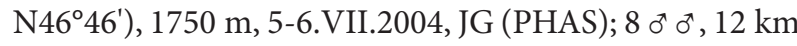
SW Araykheer, Ongij-Gol River (E102 ${ }^{\circ} 49^{\prime}$ N46 ${ }^{\circ} 22^{\prime}$ ), 1770 m, 3.VII.2004, JS (OÖLM); $2 \sigma^{\top} \sigma^{*}$, Mongol Els n.

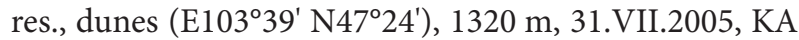
(OÖLM); $3 \sigma^{\star} \sigma^{\star}$, Arts Bogdo Ridge (E1020. $\left.8^{\prime} \mathrm{N} 44^{\circ} 65^{\prime}\right)$, 11.VII.1970, Myachmorsuren (ZISP); Selenge Aimag: $1 \sigma^{*}$, Khuder Soum, unnamed stream, $54.2 \mathrm{~km}$ E Tavin (E107¹6' N4946'), 907 m, 25.VII.2003, JG (PHAS); $2 \sigma^{\star} 0^{*}$, Khuder Soum, Khandgait Lake, 18 km SW Khuder (E107²0' N49 43'), $819 \mathrm{~m}, 23 . \mathrm{VII} .2003$, JG (PHAS); Ulaanbaatar: $2 \sigma^{\top} \sigma^{\star}$, Zuunmod env. (E106 $\left.59^{\prime} \mathrm{N} 47^{\circ} 45^{\prime}\right)$, 1630 m, 27.VII.2004, JS (OÖLM); 1 o $^{\top}$ (E106 $59^{\circ}$ N47 $\left.45^{\prime}\right)$, 14.VII.1967, VZ (ZISP); $10^{*}$, Songinyn-Amralt (E106 59' N47 $45^{\prime}$ ), 1.VII.1967, IK (ZISP); Tuv Aimag: $10^{\star}$, Batsumber Soum, Haraa Gol, $5 \mathrm{~km}$ Bayanbuural (E106 $47^{\circ}$ N4830'), $1051 \mathrm{~m}, 16 . \mathrm{VII} .2003$, JG (PHAS); 3 우 우, $20^{\wedge} 0^{\top}$, Gobi-Terelj National Park, $30 \mathrm{~km}$ E of Ulaanbataar, Tereli env. (E107 $\left.24^{\prime} \mathrm{N}^{\circ} 7^{\circ} 50^{\prime}\right), 1420 \mathrm{~m}, 13 . \mathrm{VII} .2002$, JS (PCJS); $4 \sigma^{\top} \sigma^{*}$, Gatsurt, $20 \mathrm{~km}$ E Ulaanbaatar (E107 $\left.08^{\prime} \mathrm{N} 47^{\circ} 91^{\prime}\right)$, 16.VII.1967, IK (ZISP); Dornod Aimag: $10^{\star}$, NumregiinGol, 32 km SE Salkhit Mt. (E11963' N4704'), 22-23. VII.1971, MK (ZISP); Sukhbaatar Aimag: $3 o^{\star} o^{\star}$, Dzotol (E115¹0' N4590'), 12.VII.1971, IK (ZISP); $20^{\circ} 0^{*}$, 9 km SW Dariganga (E11368' N4522'), 6.VII.1971, MK (ZISP).

Published records:

OsYTSHNJuk \& Romankova 1995: 484; KuHLMANN \& Dorn 2002: 98; ProshChalykin 2007: 879; 2012: 450; Kuhlmann 2005: 1378; 2009a: 29; Kuhlmann \& ProshCHALYKIN 2011: 19.

\section{Distribution in Mongolia:}

Usv Aimag, Khovd Aimag, *Zavkhan Aimag, Govi-Altai Aimag, Khuvsgul Aimag, Arkhangai Aimag, BayanKhongor Aimag, Uvurkhangai Aimag, ${ }^{\star}$ Selenge Aimag, Ulaanbaatar, Tuv Aimag, Dornod Aimag, ${ }^{\star}$ Sukhbaatar Aimag (Fig. 2).

\section{General distribution:}

Russia, Kazakhstan, Kyrgyzstan, Uzbekistan, Mongolia, China [Xinjiang, Qinghai, Yunnan].

\section{Colletes conradti species-group}

\section{Colletes conradti Noskiewicz 1936}

\section{Material examined:}

$\left(4 \sigma^{\star} o^{\star}\right)$ : Zavkhan Aimag: $2 \sigma^{\star} o^{\star}, 40 \mathrm{~km}$ SW Uyasutay, dunes (E96 $\left.31^{\prime} \mathrm{N}^{\circ} 7^{\circ} 31^{\prime}\right)$, 18.VII.2005, KA, JH (OÖLM); Govi-Altai Aimag: $10^{*}$, Khasagt-Khairkhan Ridge, 17 km S Dzhargalan (E95 ${ }^{\circ} 54^{\prime}$ N4650'), 1600-2100 m, 6.VI.2012, AT (IBSS); Umnugovi Aimag: $1 \sigma^{\star}$, Tost-Ula, TsaganDers-Khuduk (E100 $\left.53^{\prime} \mathrm{N}^{\circ} 3^{\circ} 28^{\prime}\right)$, 22.VI.1973, G. Medvedev (ZISP).
Published records:

Kuhlmann \& Dorn 2002: 99; Kuhlmann 2005: 1379; 2009a: 30.

\section{Distribution in Mongolia:}

Uvs Aimag, Zavkhan Aimag, Govi-Altai Aimag, Uvurkhangal Aimag, Ulaanbaatar, Tuv Aimag, Umnugovi Aimag (Fig. 2).

\section{General distribution:}

Kazakhstan, Kyrgyzstan, Mongolia, China.

\section{Colletes marginatus species-group}

\section{Colletes chengtehensis Yasumatsu 1935}

\section{Material examined:}

(16 우 우, $\left.610^{\star} \sigma^{\top}\right)$ : Usv Aimag: 2 우 우, $30 \mathrm{~km}$ NE BarunTurun (E94²4' N49³9'), 5.VII.1968, MK (ZISP); Khovd Aimag: 1 , 5 km SW Altaj-som, Bodonchin-Gol River (E92 $\left.{ }^{\circ} 5^{\prime} \mathrm{N}^{\prime} 5^{\circ} 81^{\prime}\right), 4$ 4.VIII.1968, MK (ZISP); $30^{\top} 0^{\star}$, 25 km N Bulgan, Ulyastain-Gol River (E91 ${ }^{\circ} 32^{\prime}$ N47 $09^{\prime}$ ), 31.VII.1970, MK (ZISP); $10^{\star}, 12 \mathrm{~km}$ SW Altaj-som, Bodonchin-Gol River (E92 $\left.{ }^{\circ} 5^{\prime} \mathrm{N}^{\circ} 5^{\circ} 81^{\prime}\right), 22$.VII.1970, MK (ZISP); $10^{*}, 20 \mathrm{~km}$ SE Altaj, Elkhon (E92 ${ }^{\circ} 5^{\prime}$ N45 $\left.{ }^{\circ} 81^{\prime}\right), 27$.VII.1970, VZ (ZISP); Govi-Altai Aimag: 1 ơ $^{\star}$,

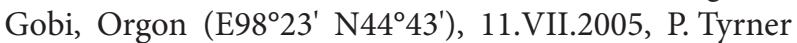
(OÖLM); Khuvsgul Aimag: $2 \sigma^{\star} \sigma^{\star}$, Buren Soum, Delger Moron Gol, 12 km W Moron (E99 59' N49³8'), 1277 m, 21.VII.2005, JG (PHAS); Arkhangai Aimag: $20^{\star} o^{\star}, 90 \mathrm{~km}$ NE Tsetserleg (E102 $\left.{ }^{\circ} 25^{\prime} \mathrm{N} 48^{\circ} 08^{\prime}\right), 1940$ m, 24.VII.2004, JS (OÖLM); $1 \sigma^{\star}, 25 \mathrm{~km}$ NE Tsetserleg (E101 ${ }^{\circ} 45^{\prime}$ N47 38'), 1730 m, 23.VII.2004, JS (OÖLM); 2 o $^{\star}$, $25 \mathrm{~km}$ W Tevshrulekh (E101 ${ }^{\circ} 75^{\prime}$ N4710'), 11.VIII.1970, Belova (ZISP); Bayan-Khongor Aimag: $10^{\star}$, Orog-Noor Lake, near Tuin-Gool River (E100 $42^{\prime}$ N45ํ3'), 5.VIII.1969, IK (ZISP); $10^{\star}$, Orog-Noor Lake (E100 $42^{\prime}$ N45 $\left.03^{\circ}\right)$, 15-16. VIII.1967, VZ (ZISP); $10^{\star}, 60 \mathrm{~km}$ SW Shine-Dzhinsta, Burkhant-Bulak (E98 $62^{\prime}$ N444ㄴ'), 29.VIII.1970, E. Narchuk (ZISP); Bulgan Aimag: $10^{\star}$, Teshig Soum, main branch of Tariankhtain Gol (E10306 ${ }^{\prime}$ N49 $\left.42^{\prime}\right), 911 \mathrm{~m}$, 10.VII.2005, JG (PHAS); 1 क , $143 \mathrm{~km} \mathrm{NE} \mathrm{Arvaykheer}$ (E10339' N47²4'), 1300 m, 26.VII.2004, JS (OÖLM); Uvurkhangai Aimag: $1 \sigma^{\star}, 137 \mathrm{~km}$ NE Arvaykheer, sandy dunes (E103 $\left.{ }^{\circ} 40^{\prime} \mathrm{N}^{\circ} 7^{\circ} 20^{\prime}\right), 1250 \mathrm{~m}, 2$ 2.VII.2004, JS (OÖLM); $10^{*}$, Tatsyn-Tsagan-Noor Lake (E101 ${ }^{\circ} 75^{\prime}$ N454ㄴ') 2-4.VIII.1969, MK (ZISP); Selenge Aimag: $10^{\star}$, Tsagaannuur Sum, SW shore Tsagaan Noor (E105 $20^{\prime}$ N49 57'), 668 m, 25.VII.2003, JG (PHAS); $10^{\star}$, EroGol River (E106 ${ }^{\circ} 1^{\prime}$ N49 $\left.72^{\prime}\right)$, 26.VII.1970, MK (ZISP); Tuv Aimag: 1 , $10^{*}, 50 \mathrm{~km}$ N Ulaanbaatar, E of Mandal (E106 $\left.46^{\prime} \mathrm{N}^{\circ} 8^{\circ} 22^{\prime}\right), 1180 \mathrm{~m}, 8$-13.VIII.2007, JH (OÖLM); 6 우 ㅇ, $75 \mathrm{~km}$ W Ulaanbaatar, dunes (E1060. N47 55'), 2.VIII.2005, JH (OÖLM); Umnugovi Aimag: 1 우, 1 o $^{\top}, 70 \mathrm{~km} \mathrm{~S}$ Saynshand (E102 $\left.33^{\circ} \mathrm{N} 42^{\circ} 55^{\prime}\right), 1100 \mathrm{~m}$, 6.VIII.2007, JH (OÖLM); Khentii Aimag: 1 ㅇ, $60^{x} o^{x}$, 
12 km N Gal-Shara (E11085' N4671'), 30.VII.1971, MK (ZISP); Dornogovi Aimag: $8 \sigma^{\star} \sigma^{\star}, 10 \mathrm{~km}$ SW Sainshand,

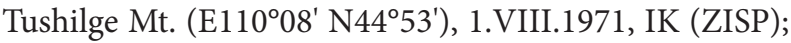
Dornod Aimag: $10^{\circ}$, Choibalsan (E114 $\left.27^{\prime} \mathrm{N} 48^{\circ} 30^{\prime}\right)$, 20.VIII.2009, YD (ISEN); $2 \sigma^{\top} \sigma^{\star}$, Steppe $\left(\mathrm{E} 115^{\circ} 19^{\prime}\right.$ N49 20'), 1.VIII.2009, YD (ISEN); $20^{\star} 0^{\star}, 32 \mathrm{~km} \mathrm{SE}$ Salkhit, Numregin-Gol River (E119 $63^{\prime}$ N47 04'), 23.VII.1971, MK, IK (ZISP); 2 ๙ $^{\star}, 75 \mathrm{~km}$ SW Salkhit, Ar-Bulak (E118³0' N4686'), 24.VII.1971, MK (ZISP); $2 o^{\top} o^{\star}, 60 \mathrm{~km}$ NE Bayan-Burd, Derkhin-Tsagan-Obo Mt. (E118 $85^{\prime}$ N47º52'), 21.VII.1971, MK (ZISP); $10^{\top}$, 20 km NW Sangin-Dalai-Noor Lake (E116 ${ }^{\circ} 55^{\prime}$ N47 $\left.7^{\circ} 10^{\prime}\right)$, 25.VII.1971, MK (ZISP); Sukhbaatar Aimag: 1 , $210 \mathrm{~km}$ SSE Baruun-Urt, steppe (E114¹0' N4530'), 29.VII.2007, $\mathrm{JH}$ (OÖLM); 2 우 ㅇ, $100 \mathrm{~km} \mathrm{SSW}$ Baruun-Urt (E112 $55^{\prime}$

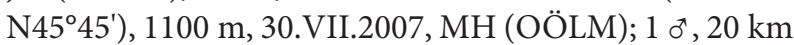
E Baruun-Urt (E11316' N4641'), 14.VII.1971, MK (ZISP); $10^{\star}, 40 \mathrm{~km}$ SE Baruun-Urt (E113 $\left.18^{\prime} \mathrm{N} 46^{\circ} 48^{\prime}\right)$, 14.VII.1971, MK (ZISP); $20^{\star} \sigma^{*}, 9 \mathrm{~km}$ SW Dariganga (E1140ㅗ' N4533'), 8.VII.1971, MK (ZISP).

Published records:

Kuhlmann \& Dorn 2002: 99; 100 (as C.pallescens Noskiewicz 1936); Kuhlmann 2005: 1379 (as C. pallescens); 2009a: 30; 2009b: 18; Kuhlmann \& ProshchaLYKIN 2011: 19; ProshChalykin \& KuhlmanN 2012: 25.

\section{Distribution in Mongolia:}

Usv Aimag, Khovd Aimag, Zavkhan Aimag, Govi-Altai Aimag, ${ }^{*}$ Khuvsgul Aimag, Arkhangai Aimag, BayanKhongor Aimag, ${ }^{\star}$ Bulgan Aimag, Uvurkhangai Aimag, *Selenge Aimag, Tuv Aimag, ${ }^{\star}$ Umnugovi Aimag, Khentii Aimag, Dornogovi Aimag, Dornod Aimag, Sukhbaatar Aimag (Fig. 2).

\section{General distribution:}

Austria, Hungary, Greece, Ukraine, Russia, Georgia, Azerbaijan, Kazakhstan, Kyrgyzstan, Turkmenistan, Uzbekistan, Iran, Mongolia, North-eastern China.

\section{Colletes succinctus species-group}

Colletes arsenjevi KunLmann 2006

Material examined:

(1 ㅇ ): Arkhangai Aimag: 1 ㅇ, Ugij-Noor Lake (E102 $46^{\prime}$ N4745'), 1.IX.1967, VZ (ZISP).

\section{Published records:}

Kuhlmann \& Dorn 2002: 100 (as C. succinctus (Linnaeus 1758)); Kuhlmann \& Quest 2006: 2; ProshchaLYKIN 2007: 878; 2012: 449; KUHLMANN 2009a: 31; 2009b: 19; Kuhlmann \& Proshchalykin 2011: 20; Niu et al. 2013: 178 .
Distribution in Mongolia:

*Arkhangai Aimag, Dornod Aimag, Sukhbaatar Aimag (Fig. 3).

\section{General distribution:}

Russia, Mongolia, China [Xinjiang, Inner Mongolia, Gansu, Shanxi].

\section{Colletes collaris Douns 1872}

Material examined:

$\left(2 \sigma^{\top} \sigma^{\top}\right)$ : Arkhangai Aimag: $2 \sigma^{\star} \sigma^{\star}$, Tsagan-Noor Lake, 18 km NE Dash-Baldoro (E9983' N48³8'), 23.VII.1975, MK (ZISP).

Published records:

Kuhlmann \& Dorn 2002: 100; Kuhlmann 2005: 1379; 2009a: 31; Proshchaly кin 2007: 878; 2012: 449; KuHLMANN \& ProshChalykin 2011: 21; NiU et al. 2013: 181.

Distribution in Mongolia:

${ }^{*}$ Arkhangai Aimag, Tuv Aimag, Khentii Aimag, Dornod Aimag, Sukhbaatar Aimag (Fig. 3).

\section{General distribution:}

Spain, France, Germany, Switzerland, Austria, Italy, Czech Republic, Hungary, Russia, Kazakhstan, Mongolia, China [Beijing, Sichuan, Xizang, Yunnan, Inner Mongolia, Heilongjiang], Japan.

\section{Species of Doubtful Placement}

\section{Colletes dorni KunLmann 2002}

Published records:

KuHlmann \& DoRn 2002: 101.

Distribution in Mongolia:

Uvs Aimag (Fig. 3).

General distribution:

Mongolia.

\section{Colletes wacki KunLmann 2002}

Material examined:

(3 우 ㅇ ): Uvurkhangai Aimag: 1 ㅇ, $12 \mathrm{~km} \mathrm{SW} \mathrm{Araykheer,}$ Ongij-Gol River (E102 ${ }^{\circ} 49^{\prime}$ N462'), 1770 m, 3.VII.2004,

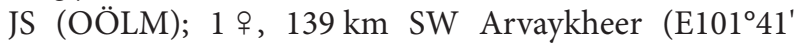
N45 ${ }^{\circ} 17^{\prime}$ ), 1430 m, 4.VII.2004, JS (RCMK); Govi-Altai

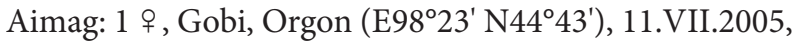
P. Tyrner (RCMK). 
Published records:

Kuhlmann \& Dorn 2002: 102; KuHLmann 2005: 1381.

\section{Distribution in Mongolia:}

Uvs Aimag, ${ }^{*}$ Uvurkhangai Aimag, ${ }^{\star}$ Govi-Altai Aimag (Fig. 2).

\section{General distribution:}

Kazakhstan, Mongolia.

\section{Acknowledgments}

We thank S. A. Belokobylskij, Yu. V. Astafurova (ZIN), V. V. Dubatolov (ISEN), J. Gelhaus (PHAS), F. Gusenleitner (OÖLM) and J. Rozen (AMNH), curators of the respective Hymenoptera collections, and A. V. Timokhov (Moscow State University, Moscow, Russia) for loans of material. Yu. N. Danilov (ISEN) kindly forwarded Colletes specimens from Russia to London. Gavin Broad, London, kindly checked the English. The work of MYP was supported by Grant of the President of the Russian Federation for young Russian scientist support (Grant number MK-411.2013.4), the Russian Funds for Basic Research (Grant numbers 12-04-31175-мол_а, 11-04-00624_a, 11-04-98585-р_восток_a), and the Far Eastern Branch of the Russian Academy of Sciences (Grant numbers 12-III-A-06-074, 12-I-П-30-03, 12-I-ОБН-02， 12-III-A-06-069，13-III-B-06-026, 13-III-Д-06-015).

\section{References}

Kuhlmann, M. 2000: Katalog der paläarktischen Arten der Bienengattung Colletes Latreille, mit Lectotypenfestlegungen, neuer Synonymie und der Beschreibung von zwei neuen Arten (Hymenoptera: Apidae: Colletinae). - Linzer biologische Beiträge 32 (1): 155-193.

Kuhlmann, M. 2002: Neue Arten der Bienengattung Colletes Latreille aus Südtibet mit Beschreibung der Route der „Deutschen Tibet Expedition Ernst Schäfer 1938/39“ (Hymenoptera: Apidae: Colletinae). - Linzer biologische Beiträge 34 (2): 1155-1178.

Kuhlmann, M. 2003: Zur Kenntnis paläarktischer Bienen der Gattung Colletes LATreille mit Beschreibung neuer Arten (Hymenoptera: Apidae: Colletinae). Linzer biologische Beiträge 35 (2): 723-746.

Kunlmann, M. 2005: Faunistik und Zoogeographie der Bienengattung Colletes LATreille 1802 (Hymenoptera: Apidae: Colletinae) in Mittelasien. - Linzer biologische Beiträge 37 (2): 1353-1396.
Kuhlmann, M. 2006: Zur Biogeographie der Seidenbienen (Hymenoptera: Colletidae: Colletes LATreILle) im Bienen-Diversitätszentrum Mittelasiens. - Mitteilungen der Deutschen Gesellschaft für Allgemeine und Angewandte Entomologie 15: 89-92.

Kuhlmann, M. 2007: Neue Colletes-Arten aus China mit Anmerkungen zu weiteren Arten (Hymenoptera: Apoidea: Colletidae). - Linzer biologische Beiträge 39 (1): 463-474.

Kuhlmann, M. 2009a: Erster Nachtrag zur Kenntnis der Bienengattung Colletes LATreille 1802 in der Mongolei mit Beschreibung einer neuen Art (Hymenoptera, Apiformes, Colletidae). - Beiträge zur Entomologie 59: 19-32.

Kuhlmann, M. 2009b: Bees of the genus Colletes (Hymenoptera, Colletidae) from Central Asia collected by the Kyushu University expeditions. - Esakia 49: 15-20.

Kuhlmann, M. 2010: Taxonomic notes on some Palaearctic Colletes bees (Insecta: Hymenoptera: Colletidae). - Linzer biologische Beiträge 42 (1): 749-755.

Kuhlmann, M.; Almeida, E. A. B.; Laurenne, N. \& Quicke, D. L J. 2009: Molecular phylogeny and historical biogeography of the bee genus Colletes Latreille, 1802 (Hymenoptera: Apiformes: Colletidae), based on mitochondrial COI and nuclear $28 \mathrm{~S}$ sequence data. - Insect Systematics and Evolution 40: 291-318.

Kuhlmann, M. \& Dorn, M. 2002: Die Bienengattung Colletes Latreille, 1802 in der Mongolei sowie Beschreibungen neuer Arten aus Sibirien und den Gebirgen Zentralasiens (Hymenoptera, Apidae, Colletinae). - Beiträge zur Entomologie 52: 85-109.

Kuhlmann, M. \& Proshchalykin, M. Yu. 2011: Bees of the genus Colletes LATREILle 1802 of the Asian part of Russia, with a key to species (Hymenoptera, Apoidea: Colletidae). - Zootaxa 3068: 1-48.

Kuhlmann, M. \& Quest, M. 2006: A new species of the bee genus Colletes Latreille, 1802 (Hymenoptera: Colletidae) from the Russian Far East and Mongolia. - Far Eastern entomologist 157: 1-4.

Michener, CH. D. 2007: The bees of the world. Second edition. - Baltimore, Maryland: The John Hopkins University Press: 953 p.

Niu, Z.-Q.; Kuhlmann, M. \& Zhu, C.-D. 2013: A review of the Colletes succinctus-group (Hymenoptera: Colletidae) from China with redescription of the male of C. gigas. - Zootaxa 3626: 173-187.

Noskiewicz, J. 1936: Die Palearktischen ColletesArten. - Prace Naukowe Wydawnictwo Towarzystwa Naukowego we Lwowie 3: 1-531.

Osytshnjuk, A. Z. \& Romankova, T. G. 1995: Family Colletidae. - In: Lehr, P. A. 1995: Key to the insects of Russian Far East. Vol. 4. Pt 1. - (Ed.). Nauka, St. Petersburg: 480-489. (In Russian). 
Proshchalykin, M. Yu. 2007: Family Colletidae. In: Lelej, A. S. 2007: Key to the insects of Russian Far East. Vol. 4. Pt 5. - (Ed.). Dalnauka, Vladivostok: 878-883. (In Russian).

Proshchalykin, M. Yu. 2012: Family Colletidae. In: LELEJ, A. S. 2012: Annotated catalogue of the insects of Russian Far East. Vol. I. Hymenoptera. - (Ed.). Dalnauka, Vladivostok: 449-451. (In Russian).
Proshchalykin, M. Yu. \& Kuhlmann, M. 2012: The bees of the genus Colletes Latreille 1802 of the Ukraine, with a key to species (Hymenoptera: Apoidea: Colletidae). - Zootaxa 3488: 1-40.

Fig. 1: Administrative map of Mongolia showing provinces (Aimag).
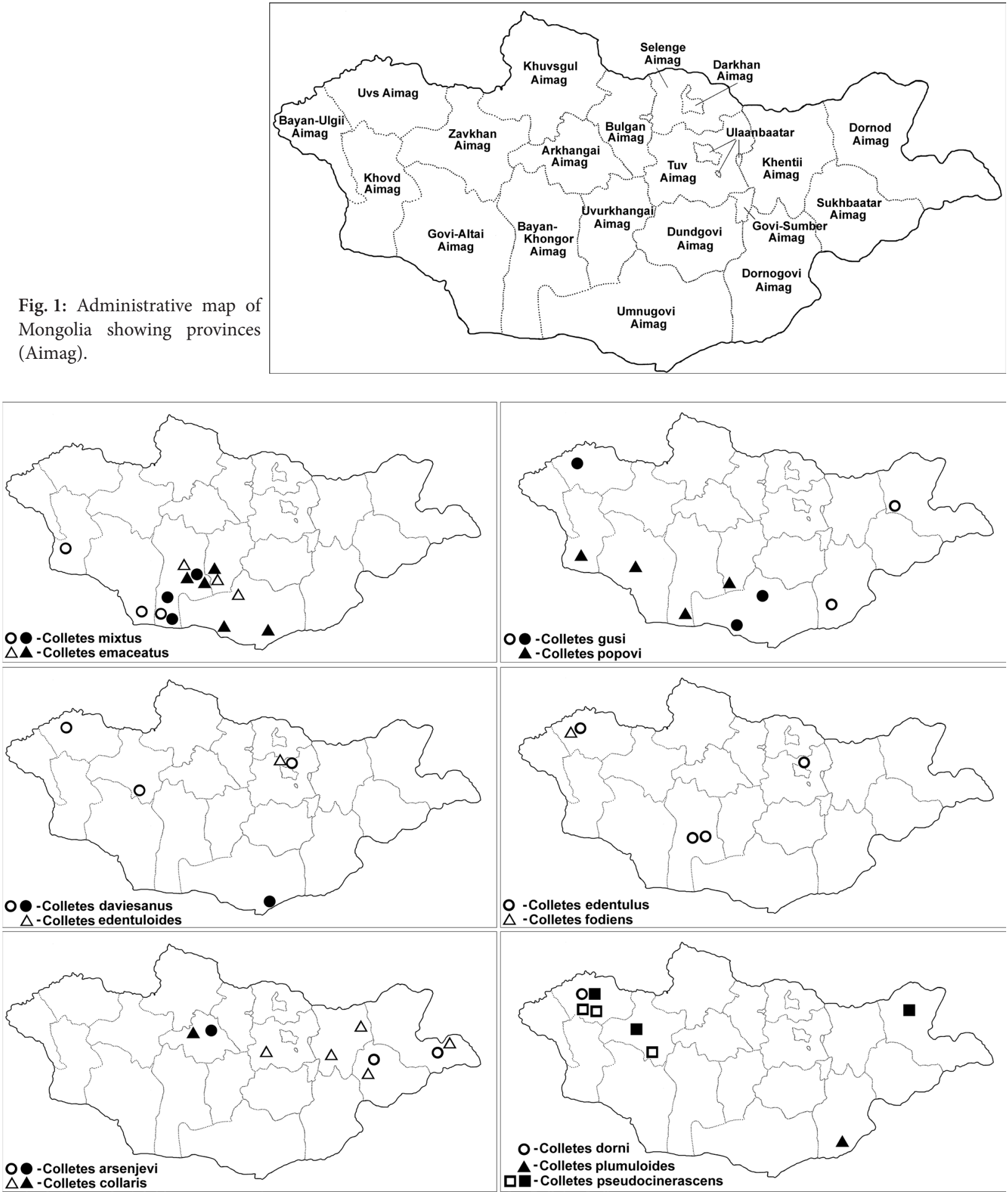

Fig. 3: Distribution of Colletes arsenjevi, C. collaris, C. daviesanus, C. dorni, C. edentuloides, C. edentulus, C. emaceatus, C. fodiens, C. gusi, C. mixtus, C. plumuloides, C. popovi and C. pseudocinerascens in Mongolia. White symbols: previous records. Filled symbols: New records. 

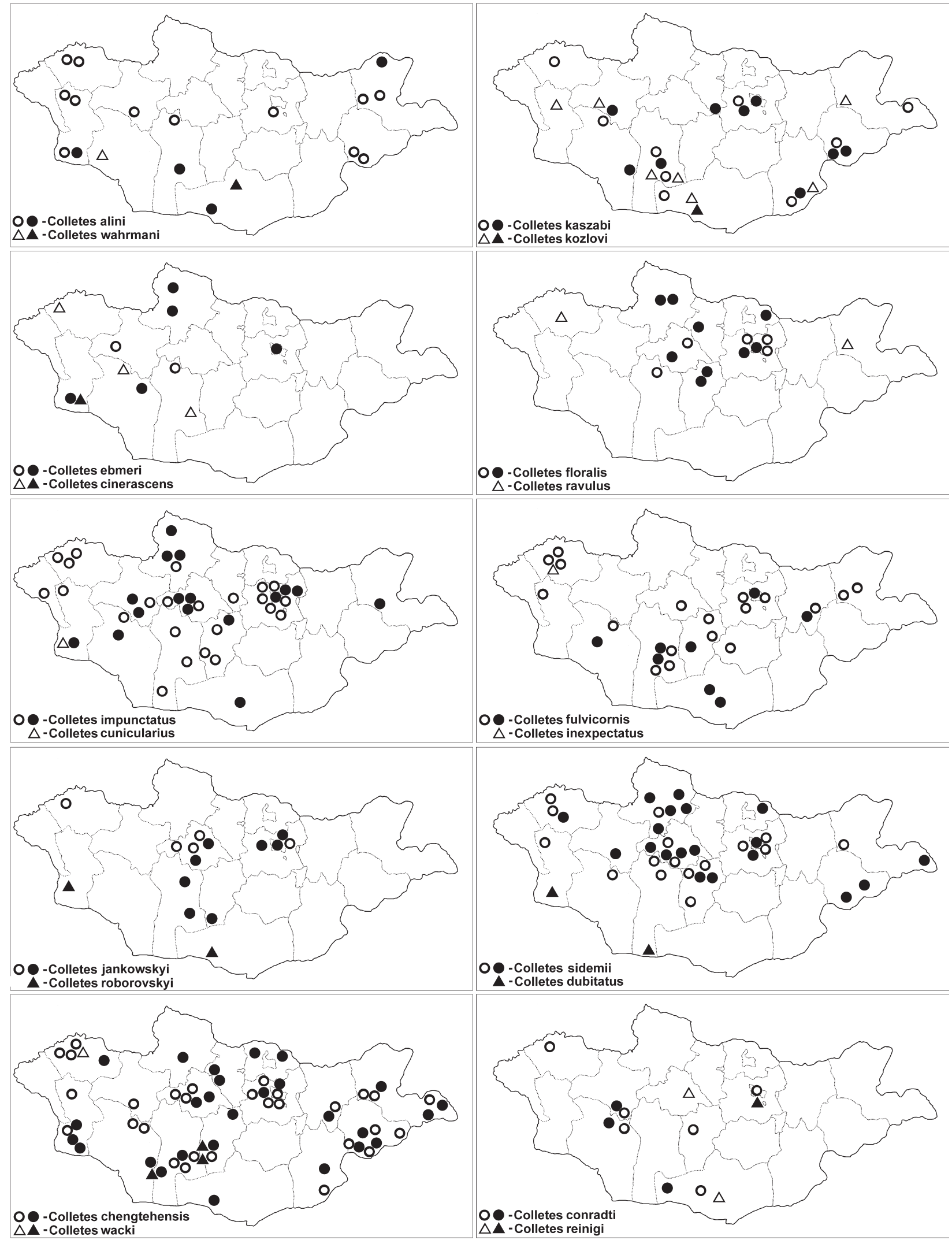

Fig. 2: Distribution of Colletes alini, C. chengtehensis, C. cinerascens, C. conradti, C. cunicularius, C. dubitatus, C. ebmeri, C. floralis, C. fulvicornis, C. impunctatus, C. inexpectatus, C. jankowskyi, C. kaszabi, C. kozlovi, C. ravulus, C. reinigi, C. roborovskyi, C. sidemii, C. wacki and C. wahrmani in Mongolia. White symbols: previous records. Filled symbols: New records. 


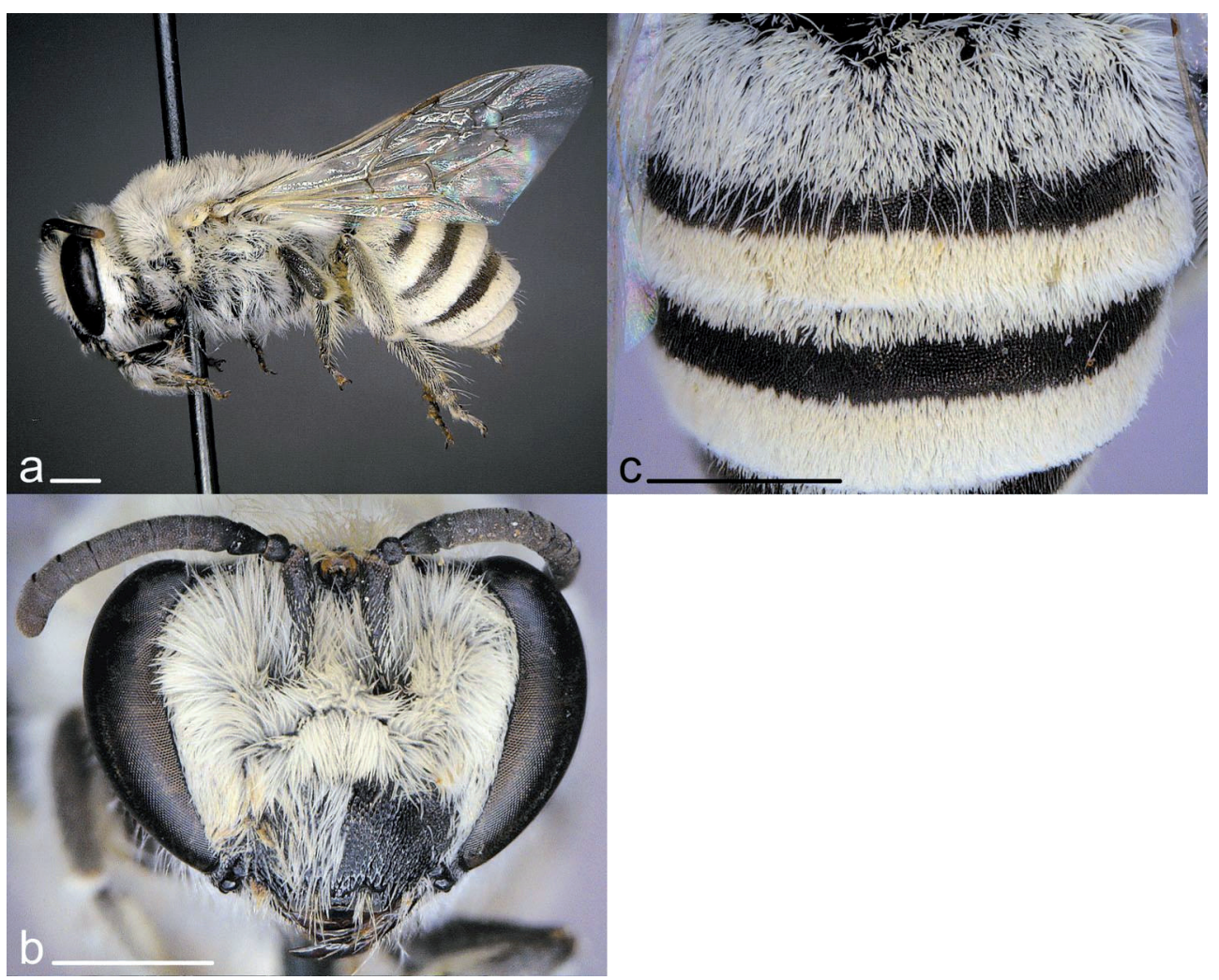

Fig. 4: Colletes dubitatus sp. n. ‥ a) Specimen in lateral view, b) head, c) metasomal terga 1 and 2. Scale bar: $1 \mathrm{~mm}$.
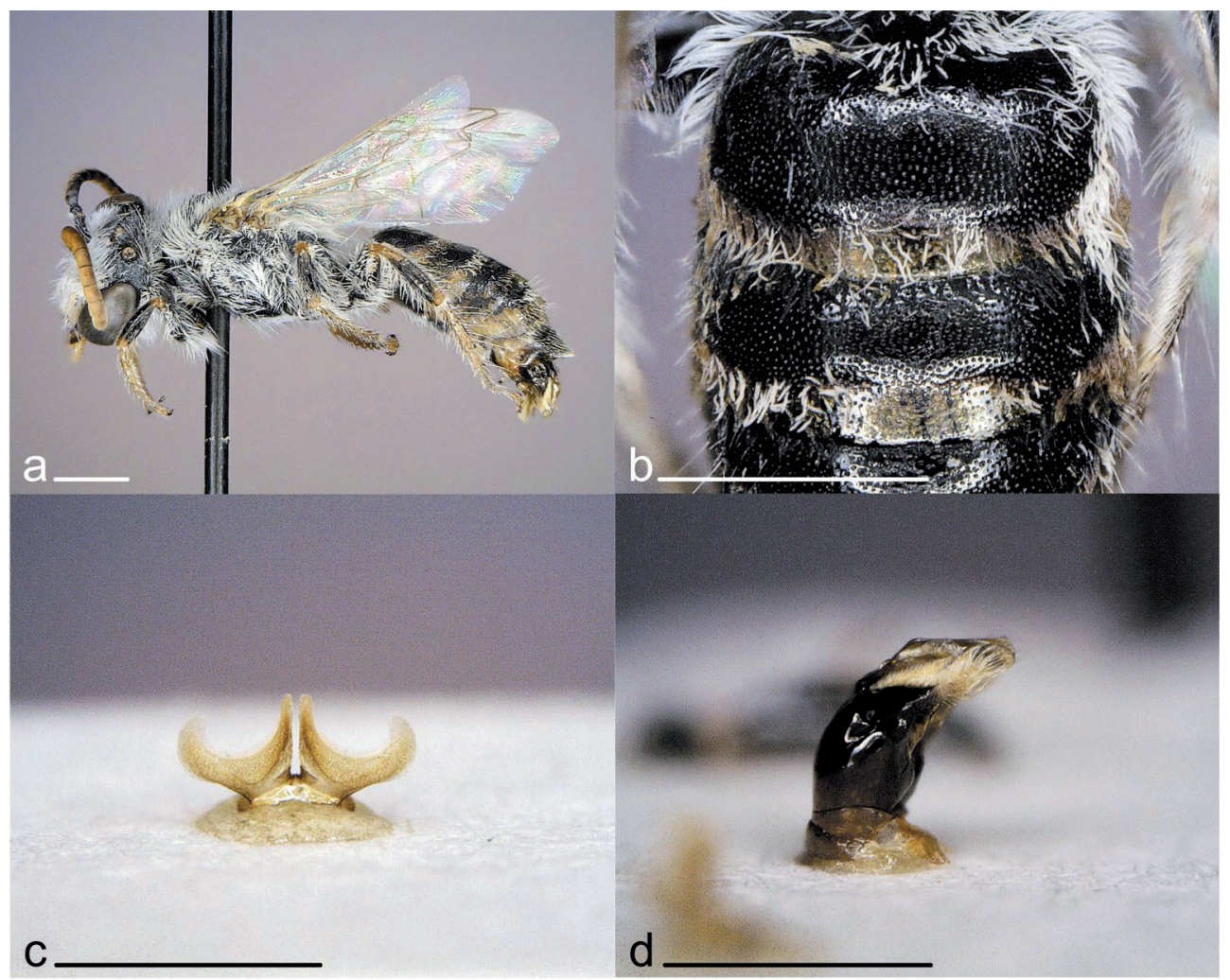

Fig. 5: Colletes plumuloides sp. n. o . a) Specimen in lateral view, b) metasomal terga 1 and 2, c) sternum $7, \mathrm{~d})$ genitalia in lateral view. Scale bar: $1 \mathrm{~mm}$. 